\title{
Resonance Raman study of the solvent dynamics for ultrafast charge transfer transition in 4-nitro-4' -dimethylamino-azobenzene
}

\author{
Nandita Biswas ${ }^{\mathrm{a})}$ and Siva Umapathy ${ }^{\mathrm{b})}$ \\ Department of Inorganic and Physical Chemistry, Indian Institute of Science, Bangalore-560012, India
}

\begin{abstract}
Contribution of solvent reorganization energy is known to be significant for ultrafast charge transfer processes, when the solvent relaxation times are slower than the rate of charge transfer. In this paper, we show that from resonance Raman intensities of a charge transfer transition in combination with Heller's time-dependent wave packet approach and Brownian oscillator model, one can have a reasonable estimate for the different types of solvent (inertial as well as diffusive) and vibrational reorganization energies. Resonance Raman spectra have been recorded for 4-nitro4 '-dimethylamino-azobenzene (DA) that undergoes photoinduced charge transfer transition, in acetonitrile and benzonitrile. In the two solvents, the total solvent reorganization energy is partitioned into its inertial and diffusive components from the available information on their relaxation time scales. Thus, partitioning of the solvent reorganization energy reveals the importance of the extent of contribution of the two components to the charge transfer rates. The short time dynamics of DA in the two solvents is then examined from a priori knowledge of the ground state normal modes in order to convert the wave packet motion in dimensionless displacements to internal coordinates. The dynamics in DA infers that within $20 \mathrm{fs}$ after photoexcitation from the ground to the charge transfer state, the excited state evolution occurs along $\mathrm{N}-\mathrm{O}, \mathrm{N}=\mathrm{N}, \mathrm{C}-\mathrm{N}$, and $\mathrm{C}-\mathrm{C}$ stretching vibrations.
\end{abstract}

\section{INTRODUCTION}

Electron transfer (ET) reactions are of fundamental importance in a variety of photophysical, photochemical, and biochemical processes, ${ }^{1,2}$ and a number of scientists have recently focused their attention on the factors controlling the rate of ET reactions. The rate of an ET reaction can be expressed as follows:

$$
k_{\mathrm{ET}}=A \exp \left\{-\frac{\left(\Delta G^{0}+\lambda\right)^{2}}{4 \lambda k_{B} T}\right\},
$$

where, $k_{B}$ is the Boltzmann constant, $k_{\mathrm{ET}}$ is the ET rate constant, $A$ is the prefactor that depends on the frequency of crossing the potential energy barrier, $\Delta G^{0}$ is the free energy change of the system undergoing ET reaction and $\lambda$ is the total reorganization energy. $\lambda$ is one of the factors that play an important role in controlling the rate of ET reactions ${ }^{1-4}$ and it includes components from the normal vibrations of the solute [vibrational/inner sphere $\left(\lambda_{V}\right)$ reorganization energy] and the polarization changes arising due to the fluctuations in the dielectric solvent environment [solvent/outer sphere $\left(\lambda_{S}\right)$ reorganization energy].

For systems undergoing photoinduced ultrafast ET reactions in solution, the solvent considerably influence the electronic structure and conformation of the solute during the ET process. ${ }^{1-3,5-13}$ Thus, the role of the solvent in the ultrafast

\footnotetext{
${ }^{a)}$ Present address: Department of Chemical Sciences, Tata Institute of Fundamental Research, Homi Bhabha Road, Colaba, Mumbai-400005, India.

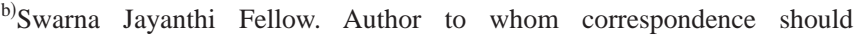
be addressed. Electronic mail: umapathy@ipc.iisc.ernet.in and suma@hamsadvani.serc.iisc.ernet.in
}

ET process have been investigated by both experimental ${ }^{10-17}$ and theoretical ${ }^{18-24}$ approaches. In particular, there have been a number of reports relating to determining the different components of the reorganization energies, namely, vibrational and solvent reorganization energies. ${ }^{25,26}$ The solvent reorganization energy, is normally determined from the line shape function of either electronic spectral response or Raman excitation profile. ${ }^{4,25}$ Interestingly, the solvent reorganization energies determined by these methods are unrealistically large ${ }^{15,16}$ even for nonpolar solvents which is in contrast to that predicted from the dielectric continuum model $^{27-29}$ and therefore its reliability has been debated. ${ }^{15}$ Thus, in this paper, we have attempted to study the various contributions to the solvent and vibrational reorganization energies for a system undergoing photoinduced ET reaction in polar solvents (acetonitrile and benzonitrile).

Resonance Raman (RR) spectroscopy is a powerful tool for extracting valuable information in the subpicosecond time scale, regarding the structure and multimode nuclear dynamics of molecules in their ground and excited electronic states. ${ }^{4,14-16,25,26,30-46}$ Thus, from an analyses of the RR intensities, it seems reasonable to consider that the most reliable determination of solvent reorganization energy is its contribution to the inertial solvation. This is particularly relevant since the RR intensities are a reflection of FranckCondon (FC) excited state dynamics. Therefore, partitioning the solvent reorganization energy $\left(\lambda_{S}\right)$ in terms of its inertial $\left(\lambda_{I}\right)$ and diffusive $\left(\lambda_{D}\right)$ components is expected to provide a realistic estimate of solvent reorganization energies at least in the case of ultrafast charge transfer (CT) process, where the ET rates are considerably faster than the solvent relax- 
ation time. The inertial and diffusive reorganization energies thus, reflect distinct phases of solvent relaxation (first, the ultrafast inertial component due to the librational motion of the solvent molecules, characterized by the time constant, $\tau_{I}$ and the second, a slow component which arises due to the diffusive motion of the solvent, with time constant, $\tau_{D}$ ). Evidence for the existence of the inertial component has been obtained experimentally from the time-dependent fluorescence Stokes shift (TDFSS), 5,12,47-49 stimulated photon echo $^{50}$ as well as femtosecond infrared (IR) ${ }^{51}$ and absorption spectroscopy. ${ }^{3}$ It has also been confirmed by extensive theoretical studies ${ }^{24,50,52}$ during the past. The caveat in such an approach involving RR intensities has always been the lack of knowledge of the low frequency intramolecular vibrational and solvent contributions to the reorganization energies. ${ }^{4}$ However, in the present study, we find that it is feasible to calculate the different contributions to the reorganization energies, as discussed below (vide infra).

During the past, RR technique has been utilized to study

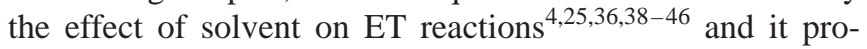
vided a good estimate of total solvent and vibrational reorganization energy terms which are very sensitive to the conformational and geometrical changes of the molecular system, undergoing photo induced CT transition. The normal vibrations that are coupled to the resonant electronic transition appear intense in the RR spectrum and its intensity reflects the strength of coupling and the dynamics that occur in the excited electronic state. Previous calculations of RR intensities with the inclusion of solvent effects ${ }^{4,25}$ have assumed single exponential energy relaxation (Markovian dynamics) which does not hold good in the subpicosecond time scales for fast solvents (such as, acetonitrile, methanol and water). Under such time scales, the overdamped Brownian oscillator model, which assumes a Gaussian-Markovian description for the correlation function of the solvent induced shifts of the solute energy levels, can be generalized to include the non-Markovian dynamics (multiexponential in time $)^{24}$ or, contribution from the ultrafast inertial response ${ }^{53}$ in addition to the slow (diffusive) Markovian dynamics. This approach is expected to provide information on the reorganization energy contribution to a ET process for both inertial and diffusive solvation dynamics.

In this paper, in an attempt to quantitatively understand the extent of contribution of various internal vibrations and solvent to the total reorganization energy, we have chosen to study a photochemical donor-acceptor system 4-nitro, $4^{\prime}$-dimethylamino-azobenzene (DA) which upon excitation from the ground to the excited CT state undergoes an increase in dipole moment by 10.27 Debye ${ }^{54}$ and it exhibits a strong CT absorption in the visible region and does not fluoresce at room temperature (RT). Hence, DA is a very useful probe for the study of intramolecular ET reaction. Thus, we have carried out a detailed experimental and theoretical investigation on the RR intensity analysis of the intramolecular CT transition of DA in two solvents of different polarity [viz. acetonitrile (0.713) and benzonitrile (0.904)] using excitation wavelengths, ranging from $450-550 \mathrm{~nm}$ in resonance with the lowest excited singlet electronic state. The polarity of these solvents have been expressed in terms of the $\pi^{*}$ scale developed by Kamlet, Abbout and Taft, ${ }^{55}$ which is a measure of solvent dipolarity and polarizability. An important point to be noted here is that the solvents, acetonitrile and benzonitrile have been chosen in such a way that they differ in their relaxation time and viscous property. Benzonitrile is more viscous (1.267 centipoise) than acetonitrile (0.369 centipoise), as a result of which they have widely different relaxation behavior. ${ }^{51,56}$ Here, we also report a novel approach of partitioning the total solvent reorganization energy $\left(\lambda_{S}\right)$ into its inertial $\left(\lambda_{I}\right)$ and diffusive $\left(\lambda_{D}\right)$ components for an ultrafast ET reaction from the available information on the solvent relaxation time scales. The parameters viz. vibrational and solvent reorganization energies associated with the CT transition have been elucidated from the modeling of RR intensities as well as the absorption spectra using Heller's time-dependent wave packet approach with the inclusion of solvent effects (overdamped Brownian oscillator model ${ }^{18,53}$ for diffusive solvent relaxation and underdamped Brownian oscillator model ${ }^{53}$ for inertial solvent relaxation). Combining the results obtained from Heller's time-dependent wave packet analysis of the experimental RR intensities and the knowledge of normal mode descriptions, it has been possible to elucidate the ultrafast dynamical information along various vibrational coordinates of DA in acetonitrile and benzonitrile.

\section{EXPERIMENTAL METHODS}

The excitation wavelengths for the resonance Raman experiments in the visible region viz. 450 to $550 \mathrm{~nm}$ were scanned using a tunable laser pulse output from an optical parametric oscillator (OPO) (MOPO 730, Spectra Physics). The OPO was pumped by the third harmonic $(355 \mathrm{~nm})$ of a $10 \mathrm{~Hz} Q$-switched, Nd-YAG laser (GCR 250, Spectra Physics) providing a high-energy laser pulse of $560 \mathrm{~mJ}$. The energy of the OPO output was in the range of 4-8 $\mathrm{mJ} / \mathrm{pulse}$. The energy of the laser beam near the sample is about 15$16 \%$ less than the energy mentioned above. The detailed experimental setup is given in Ref. 37(c). A SPEX 1404 double monochromator was used with two 600 groove gratings blazed at $500 \mathrm{~nm}$ to disperse the scattered light. A liquid nitrogen cooled charge coupled device (Princeton Instruments) with $576 \times 378$ pixels was used as the multichannel detector. The recorded Raman spectra were calibrated using known solvent bands as reference and the spectral resolution is estimated to be $5 \mathrm{~cm}^{-1}$.

4-nitro, $4^{\prime}$-dimethylamino-azobenzene (DA) used in the Raman experiments was prepared by the coupling reaction of $p$-nitrobenzene diazonium tetrafluoroborate with $\mathrm{N}, \mathrm{N}$ dimethyl aniline at $3-5{ }^{\circ} \mathrm{C}^{57}$ and was purified by column chromatography, on acidic alumina with toluene as eluent followed by recrystallization from toluene. ${ }^{57}$ Acetonitrile $\left(\mathrm{CH}_{3} \mathrm{CN}\right)$ was of analytical grade and was used as received. Benzonitrile $\left(\mathrm{C}_{6} \mathrm{H}_{5} \mathrm{CN}\right)$ was purchased from Aldrich Chemical Company and used as received. Freshly prepared sample solutions of DA in acetonitrile and benzonitrile were circulated through a capillary at the rate of about $10 \mathrm{ml} / \mathrm{min}$. These solutions were used in the dark for each Raman measurement. In DA, since the excited state is short lived, during a nanosecond laser pulse the molecule might photoisomerize. 
The amount of the cis isomer in the solution, which is produced by photoisomerization, is negligibly small because the cis isomer thermally returns to the trans form with a time constant as short as tens of microseconds. Hence, the irradiated solution of DA mainly consisted of the trans isomer. This was checked routinely after each Raman measurement by UV-visible absorption as well as vibrational spectroscopy, since the cis and trans isomers have distinguishable absorption as well as vibrational spectra. During the Raman measurements, since it was observed that tightly focusing the laser beam onto the sample led to a change in color of the solution (viz. high laser energy was leading to photodegradation of the sample), we used a long focal length lens of about $50 \mathrm{~cm}$, so that the beam diameter at the irradiated volume was about $2.5 \mathrm{~mm}$. Under these conditions, it is observed that both the Raman spectra and the absolute scattering intensity are independent at the laser pulse energy indicating the absence of any nonlinear effect.

The reabsorption of the resonance Raman scattered light by the sample was minimized by using a $180^{\circ}$ backscattering collection geometry. Remaining reabsorption of the Raman scattered light by the sample were corrected using the methods described previously. ${ }^{33(d), 36,58}$ The sample concentrations used for Raman experiments were $0.71 \times 10^{-3} \mathrm{M}$ in acetonitrile and $2.1 \times 10^{-3} \mathrm{M}$ in benzonitrile. The absolute Raman cross section of benzonitrile, $1000 \mathrm{~cm}^{-1}$ band was obtained from the integrated Raman intensity ratio relative to acetonitrile, $918 \mathrm{~cm}^{-1}$ peak as reported previously. ${ }^{59,60}$ Thus, the absolute Raman cross sections for DA in acetonitrile and benzonitrile were determined relative to the absolute intensities of the respective solvent bands $\left(918 \mathrm{~cm}^{-1}\right.$ for acetonitrile and $1000 \mathrm{~cm}^{-1}$ for benzonitrile).

\section{THEORETICAL AND COMPUTATIONAL METHODS}

The main objective of the data analysis procedure is to partition the total solvent reorganization energy into inertial as well as diffusive reorganization energy terms. In addition, we are interested in determining the mode-specific vibrational reorganization energies and the short time dynamics that depend on the displacement of the excited state potential energy minimum with respect to the ground state, along each vibrational coordinate. Initial estimates of the geometry change along each Raman active vibration following photoexcitation are obtained from the experimental resonance Raman intensities assuming short time dynamics (i.e., Raman intensity, $I_{R} \propto \Delta_{v}^{2} \omega_{v}^{2}$ ) and this assumption is reasonable since our interest lies only in the Franck-Condon region of the total potential energy surface. These geometry changes are then refined by direct computation of the absorption and Raman cross section from the first principles via the timedependent theory of Raman scattering pioneered by Heller et $a l .{ }^{61}$ In the time-dependent approach, the resonance Raman cross section ${ }^{61(a)} \sigma_{R, i \rightarrow f}$ for the transition from ground electronic ground vibrational state $|i\rangle$ to the ground electronic excited vibrational state $|f\rangle$, in an isolated polyatomic molecule under the Born-Oppenheimer as well as Condon approximation, can be expressed as a half Fourier transform of the correlation function $\langle f \mid i(t)\rangle,{ }^{14,26,31,32,38}$

$$
\begin{aligned}
\sigma_{R, i \rightarrow f}\left(E_{L}\right)= & \frac{8 \pi e^{4} M^{4} E_{S}^{3} E_{L}}{9 \hbar^{6} c^{4}} \\
& \times\left|\int_{0}^{\infty}\langle f \mid i(t)\rangle \exp \left[\frac{i\left(E_{L}+E_{i}\right) t}{\hbar}-\frac{\Gamma t}{\hbar}\right] d t\right|^{2},
\end{aligned}
$$

where, $E_{S}$ and $E_{L}$ are the energies of the scattered and incident photons, $M$ is the electronic transition moment, $E_{i}$ is the zero point energy of the ground electronic state, $\Gamma$ is the homogeneous linewidth, $e$ is the charge of the electron, $c$ is the velocity of light and $\hbar=h / 2 \pi$ ( $h$ being Planck's constant) and $|i(t)\rangle$ is the evolving wave packet on the excited electronic surface, at various intervals of time, under the influence of the excited state Hamiltonian, $H_{\mathrm{ex}}$,

$$
|i(t)\rangle=\exp \left(-\frac{i H_{\mathrm{ex}} t}{\hbar}\right)|i\rangle,
$$

where, $\exp \left(-i H_{\mathrm{ex}} t / \hbar\right)$ is the time-evolution operator. The time evolution of the wave packet is carried out using the grid technique. $^{62,63}$

Similarly, the expression for the absorption cross section $^{61(\mathrm{~b}), 61(\mathrm{e})}$ in the time-dependent formalism is expressed as full Fourier transform of the autocorrelation function $\langle i \mid i(t)\rangle$,

$$
\begin{aligned}
\sigma_{A}\left(E_{L}\right)= & \frac{4 \pi e^{2} M^{2} E_{L}}{6 \hbar^{2} c n} \int_{-\infty}^{\infty}\langle i \mid i(t)\rangle \\
& \times \exp \left[\frac{i\left(E_{L}+E_{i}\right) t}{\hbar}-\frac{\Gamma|t|}{\hbar}\right] d t,
\end{aligned}
$$

where, $n$ is the refractive index of the solution.

However, in solution, the solute molecules are subjected to random forces due to their interaction with the surrounding solvent molecules, leading to significant line broadening and hence loss of structures in the spectra. Thus, the resonance Raman intensities are analyzed on the basis of Brownian oscillator model developed by Mukamel and co-workers ${ }^{14(b), 18,53}$ where the solvent coordinates are treated as displaced harmonic oscillators that are frictionally damped to different extent. In this model, three parameters (frequency, coupling strength, and friction coefficient) are required in order to represent each oscillator. Here, we consider the case of frictionally underdamped as well as overdamped Brownian oscillator where all the solvent modes are grouped into one effective mode and the solvent response can be expressed in terms of the parameters, solvent reorganization energy, $\lambda$ and frequency of the oscillator, $\omega$ (or, the inverse time scale of fluctuation, $\Lambda$ ). Due to the solutesolvent interaction, the electronic energy levels fluctuate with time and the resonance Raman cross section for the transition $|\mathbf{i}\rangle \rightarrow|\mathbf{f}\rangle$ is given by ${ }^{14,18}$ 


$$
\begin{aligned}
\sigma_{R, i \rightarrow f}\left(E_{L}\right)= & \frac{8 \pi e^{4} E_{S}^{3} E_{L} M^{4}}{9 \hbar^{6} c^{4}} \\
& \times \sum_{i} P_{i} \mid \int_{0}^{\infty} d t\langle f \mid i(t)\rangle \\
& \times\left.\exp \left[i\left(E_{L}+E_{i}\right) \frac{t}{\hbar}-\frac{\Gamma t}{\hbar}-g_{\text {solv }}(t)\right]\right|^{2},
\end{aligned}
$$

where, $P_{i}$ is the Boltzmann population of state $|i\rangle$ and the dynamic contribution of the solvent environment treated as a frictionally damped oscillator is expressed by $\exp \left[-g_{\text {solv }}(t)\right]$, where the function, $g_{\text {solv }}(t)$ is expressed as follows:

$$
g_{\text {solv }}(t)=\int_{0}^{t} d \tau_{2} \int_{0}^{\tau_{2}} d \tau_{1} C\left(\tau_{1}\right),
$$

$C\left(\tau_{1}\right)$ being the correlation function of the electronic energy gap $\partial E_{0}$ and is given by

$$
C\left(\tau_{1}\right) \equiv \frac{1}{\hbar^{2}}\left\langle\partial E_{0}\left(\tau_{1}\right) \partial E_{0}(0)\right\rangle .
$$

Thus, the general solution for $g_{\text {solv }}(t)$ (in the overdamped limit) consists of both real and imaginary terms which is given as follows: ${ }^{18}$

$$
g_{\text {diff }}(t)=g_{R}(t)+i g_{I}(t),
$$

where

$$
\begin{aligned}
g_{I}(t)= & -\frac{\lambda_{D}}{\Lambda}[\exp (-\Lambda t)+\Lambda t-1], \\
g_{R}(t)= & \left(\frac{\lambda_{D}}{\Lambda}\right) \cot \left(\frac{\hbar \Lambda}{2 k_{B} T}\right)[\exp (-\Lambda t)+\Lambda t-1] \\
& +\frac{4 \lambda_{D} \Lambda k_{B} T}{\hbar} \sum_{n=1}^{\infty} \frac{\exp \left(-\nu_{n} t\right)+\nu_{n} t-1}{\nu_{n}\left(\nu_{n}^{2}-\Lambda^{2}\right)},
\end{aligned}
$$

where

$$
\nu_{n}=2 \pi n k_{B} T / \hbar \text {. }
$$

In order to derive the $g_{\text {solv }}(t)$ expression to incorporate the inertial reorganization energy component, we invoke the solvation time correlation function in the underdamped coherent motion limit. ${ }^{53}$ The existence of the large amplitude, rapid inertial Gaussian component in the solvent response can be described by the underdamped Brownian oscillator model. Therefore, the general solution for $g_{\text {solv }}(t)$ (in the underdamped coherent motion limit) can be written as ${ }^{53}$

$g_{\text {iner }}(t)=\frac{\lambda_{l}}{\omega}[1-\cos (\omega t)] \operatorname{coth}\left(\frac{\hbar \omega}{2 k_{B} T}\right)-\frac{i \lambda_{I}}{\omega}[\omega t-\sin (\omega t)]$,

where, $\lambda_{I}$ and $\omega$ correspond to the fast inertial reorganization energy and the oscillator frequency, respectively. Thus, the solution to the dynamic contribution of the solvent environment $g_{\text {solv }}(t)$ having contributions from the fast inertial and slow diffusive solvent response is expressed as

$$
g_{\text {solv }}(t)=g_{\text {iner }}(t)+g_{\text {diff }}(t),
$$

where $g_{\text {iner }}(t)$ and $g_{\text {diff }}(t)$ correspond to the fast inertial and slow diffusive solvent response and are expressed by Eqs. (12) and (8)-(11), respectively.

The absorption cross section, for a molecule in condensed phase, in the time-dependent formalism is expressed as follows:

$$
\begin{aligned}
\sigma_{A}\left(E_{L}\right)= & \frac{4 \pi e^{2} E_{L} M^{2}}{3 \hbar^{2} c n} \sum_{i} P_{i} \operatorname{Re} \int_{0}^{\infty} d t\langle i \mid i(t)\rangle \\
& \times \exp \left[i\left(E_{L}+E_{i}\right) \frac{t}{\hbar}-\frac{\Gamma t}{\hbar}-g_{\text {solv }}(t)\right] .
\end{aligned}
$$

For the time-dependent calculations it has been assumed that there is no coordinate dependence of the transition length and no change in the normal coordinate on electronic excitation (no Duschinsky rotation). Moreover, the excited state potential surfaces are harmonic, with no change in frequencies and differ from the ground state surfaces by only a constant term in energy (zero-zero energy, $E_{0}$ ) and are displaced by an amount $\Delta_{v}$ (in dimensionless units) with respect to the ground state minimum. The dimensionless displacements for the respective modes are obtained at all the excitation wavelengths from the experimental Raman intensities relative to the intensity for the 1593 and $1587 \mathrm{~cm}^{-1}$ band in acetonitrile and benzonitrile, respectively. The average dimensionless displacement for the respective Raman active mode is then calculated and fed into our simulations for the absorption spectrum and the Raman excitation profiles. This procedure is repeated several times for various values of dimensionless displacements in order to get minimum deviation for the simulated absorption spectrum and the Raman excitation profiles in comparison to the respective experimental spectrum. Thus, the best fit value for the displacement, $\Delta_{v}$ corresponding to each Raman active vibration is obtained. The final values of the geometry change $\Delta_{v}$ (in dimensionless coordinates) obtained from these calculations are normally used to obtain the mode-specific vibrational reorganization energies, $\lambda_{v}$ using the following expression:

$$
\lambda_{v}=\frac{\omega_{v} \Delta_{v}^{2}}{2}
$$

where $\omega_{v}$ corresponds to the vibrational frequency of the Franck-Condon active vibrational modes.

After photoexcitation, the center of the wave packet undergoing separable harmonic motion at time, $t$ is expressed in dimensionless normal coordinates ${ }^{14(\mathrm{~d}), 14(\mathrm{e})}$ as

$$
q_{\alpha}(t)=\Delta_{\alpha}\left(1-\cos \omega_{\alpha} t\right)
$$

where $q_{\alpha}=0$ for all the normal modes $\alpha$ at zero time when the molecule is at ground state equilibrium position and $\omega_{\alpha}\left(=\bar{\omega}_{\alpha} / 5308.8, \bar{\omega}_{\alpha}\right.$ is the frequency in $\mathrm{cm}^{-1}$ and 5308.8 corresponds to the value of $\hbar$ in units of $\mathrm{cm}^{-1}$ fs is the vibrational frequency in $\mathrm{fs}^{-1}$. In order to visualize the short time dynamics in terms of internal coordinate changes, the dimensionless normal coordinate displacement, $\left\{q_{\alpha}(t)\right\}$ is converted to the internal coordinate displacement $\left\{S_{i}(t)\right\}$ by the following expression: ${ }^{14(d), 14(e)}$ 

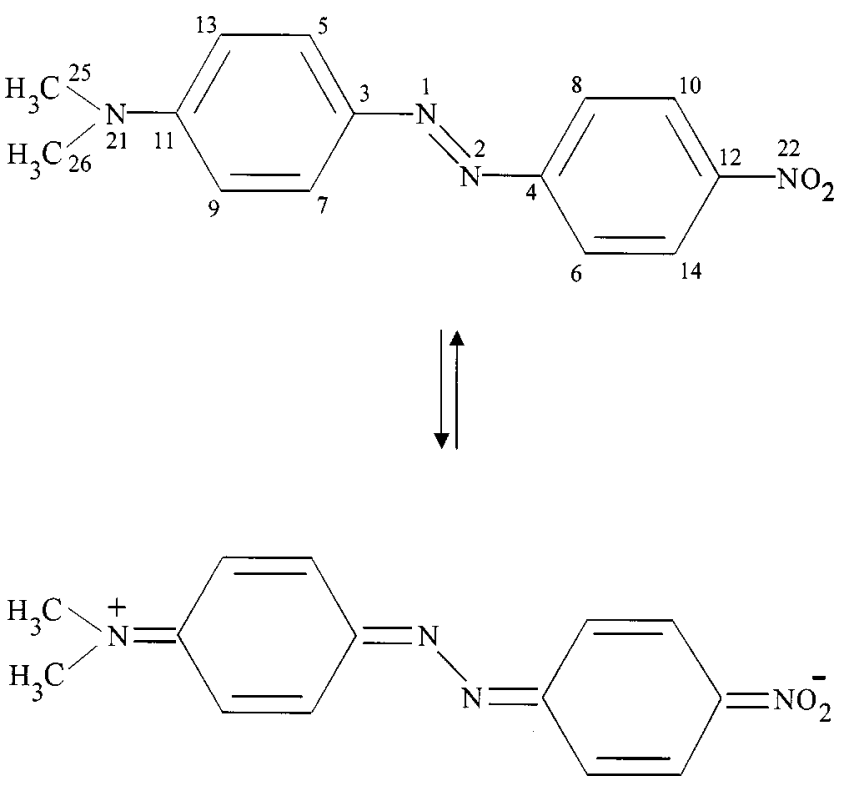

FIG. 1. Neutral and zwitterionic structures of 4-nitro, $4^{\prime}$-dimethylaminoazobenzene (DA) with atom numbering on heavy atoms.

$$
S_{i}(t)=\left(\frac{\hbar}{c}\right)^{1 / 2} \sum_{\alpha} A_{\alpha i} \bar{\omega}_{\alpha}^{-1 / 2} q_{\alpha}(t),
$$

where $S_{i}(t)$ is the change in internal coordinates viz. stretches, bends, torsions, wags, etc. (as defined by Wilson, Decius, and $\mathrm{Cross}^{64}$ ) at time, $t$, with respect to the ground state equilibrium geometry, $A_{\alpha i}$ is the normal mode coefficient $\left(\delta S_{i} / \delta Q_{\alpha}\right)$ and is obtained from normal coordinate analysis $\left(Q_{\alpha}\right.$ being the normal coordinate) via the unitary transformation, ${ }^{34,36}$

$$
A F A^{+}=\Lambda,
$$

where the matrix, $A$ represents the normal mode vectors, and the diagonal matrix, $\Lambda$ represents the eigenvalues. The potential energy matrix, $F$ is obtained from the valence force field $^{65}$ at the ground state equilibrium geometry for 4-nitro, $4^{\prime}$-dimethylamino-azobenzene which is computed employing the density functional method, B3LYP with $6-31 \mathrm{G}$ basis set using the GAUSSIAN 94 package. ${ }^{66}$ B3LYP with $6-31 \mathrm{G}$ is found to be very accurate in predicting vibrational frequencies, and therefore, the force field for polyatomic systems. ${ }^{65,67}$

\section{RESULTS}

4-nitro, $4^{\prime}$-dimethylamino-azobenzene (DA) undergoes photoinduced intramolecular electron transfer in the singlet excited state and exhibits a distorted structure $\left(C_{1}\right.$ symmetry) in solution. The neutral as well as the zwitterionic structures of DA are shown in Fig. 1. DA is characterized by the presence of lowest energy charge transfer (CT) transition. On resonance excitation to this charge transfer transition, Raman bands are observed for the vibrational modes that are Franck-Condon active for this transition. Hence, a detailed analysis of the experimental and simulated absorption spectra as well as Raman excitation profiles REPs and the short

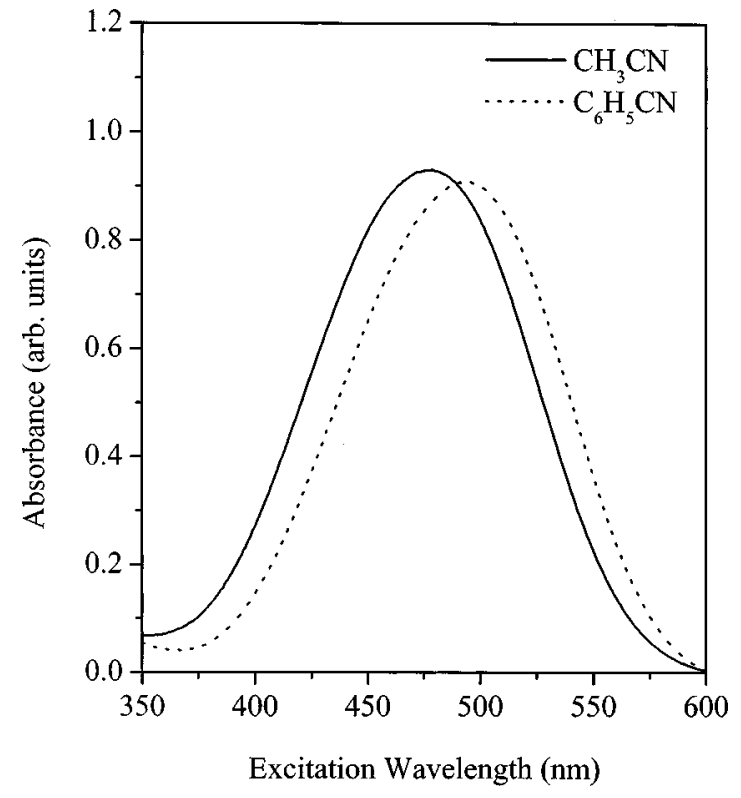

FIG. 2. Absorption spectra of DA in acetonitrile and benzonitrile.

time dynamics of DA in solvents of different polarity have been carried out and are presented in the following sections.

\section{Absorption spectra}

Electron transfer in the first excited singlet state $S_{1}$ is manifested in the static absorption spectrum which is sensitive to the Kamlet, Abbout, and Taft polarity parameter $\left(\pi^{*}\right)$ of the solvent. The absorption spectra of DA in acetonitrile $\left(\mathrm{CH}_{3} \mathrm{CN}\right)$ and benzonitrile $\left(\mathrm{C}_{6} \mathrm{H}_{5} \mathrm{CN}\right)$ are shown in Fig. 2. The absorption spectra as shown in Fig. 2 are strongly dependent on the solvent polarity and the absorption maxima $\left(\lambda_{\max }\right)$ undergo a bathochromic shift from $479 \mathrm{~nm}$ in acetonitrile to $496 \mathrm{~nm}$ in benzonitrile as the solvent polarity $\left(\pi^{*}\right)$ increases from 0.713 (acetonitrile) to 0.904 (benzonitrile). This large shift of $17 \mathrm{~nm}$ in the absorption maxima with increase in solvent polarity is attributed to the net stabilization of the charge separated Franck-Condon excited state $\left(S_{1}\right)$ relative to the ground state.

\section{Resonance Raman spectra}

The Raman spectra of DA in acetonitrile have been measured in resonance with the charge transfer transition at the excitation wavelengths, 450, 460, 470, 480, 490, 500, 510, 520, 530, 540, and $550 \mathrm{~nm}$. In Fig. 3 are shown the solvent subtracted RR spectra of DA in acetonitrile at 450, 480, 500, 530 , and $550 \mathrm{~nm}$. The Raman fundamentals of DA in acetonitrile are observed at 1104, 1138, 1199, 1344, 1398, 1428, 1453 , and $1593 \mathrm{~cm}^{-1}$. The modes that undergo maximum distortion on Franck-Condon excitation are expected to be most intense in the RR spectra. It is observed from the figure that the most intense Raman bands correspond to $\mathrm{N}-\mathrm{O}$ and $\mathrm{N}=\mathrm{N}$ stretches appearing at 1344 and $1398 \mathrm{~cm}^{-1}$, respectively, followed by 1428, 1453, 1138, 1104, and $1593 \mathrm{~cm}^{-1}$. A weak band is observed at $1199 \mathrm{~cm}^{-1}$. The intensity patterns at all the excitation wavelengths are almost similar. At each wavelength, the absolute Raman cross section for the 


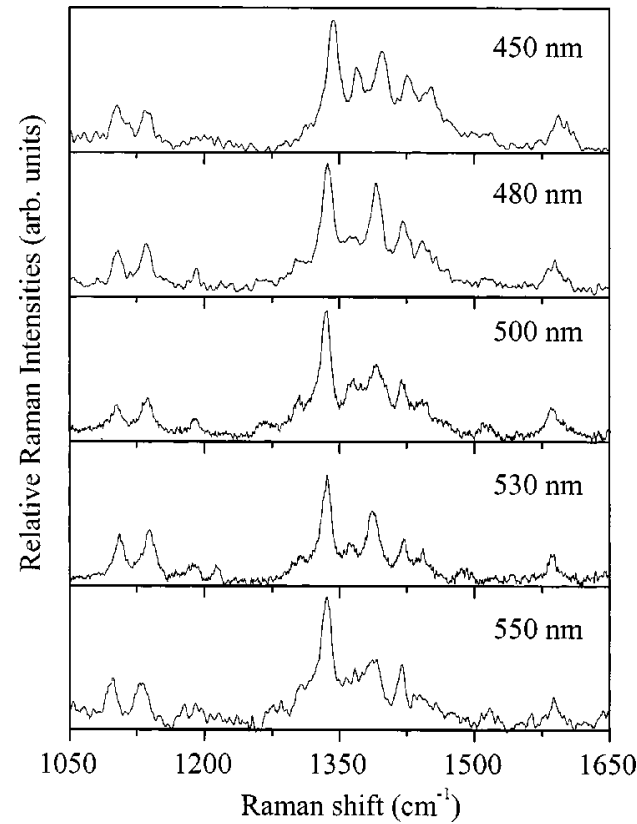

FIG. 3. Resonance Raman spectra of DA in acetonitrile at excitation wavelengths, $450,480,500,530$, and $550 \mathrm{~nm}$.

fundamentals of DA in acetonitrile has been determined with respect to the solvent band appearing at $918 \mathrm{~cm}^{-1}$.

The Raman spectra of DA in benzonitrile have also been measured in resonance with the CT transition at the excitation wavelengths, 450, 460, 470, 480, 490, 500, 510, 520, 530, 540, and $550 \mathrm{~nm}$. In Fig. 4 are shown the solvent subtracted RR spectra of DA in benzonitrile at excitation wavelengths, 450, 480, 500, 530, and $550 \mathrm{~nm}$. The resonance Raman spectra of DA in benzonitrile have various fundamental peaks appearing at 1106, 1140, 1195, 1336, 1393, 1422,1447 , and $1587 \mathrm{~cm}^{-1}$. From a comparison of Figs. 3

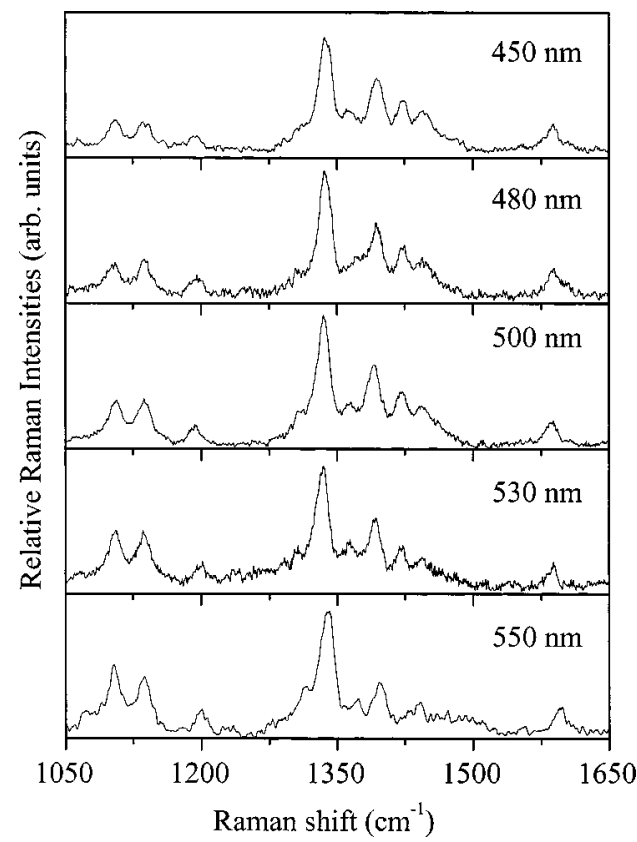

FIG. 4. Resonance Raman spectra of DA in benzonitrile at excitation wavelengths, $450,480,500,530$, and $550 \mathrm{~nm}$. and 4 , it is observed that the intensity patterns for the vibrational modes in acetonitrile and benzonitrile are very similar, with the $\mathrm{N}-\mathrm{O}$ stretch appearing at $1336 \mathrm{~cm}^{-1}$ being the most intense Raman band, followed by 1393 and $1422 \mathrm{~cm}^{-1}$ corresponding to $\mathrm{N}=\mathrm{N}$ stretch and $\mathrm{C}-\mathrm{C}$ stretch, respectively. A few more bands of moderate intensity appear at 1106, 1140, 1447 , and $1587 \mathrm{~cm}^{-1}$ followed by a weak band at 1195 $\mathrm{cm}^{-1}$. Moreover, the effect of solvent polarity on the vibrational frequencies have been found to be negligible. At each wavelength, the absolute Raman cross section of the fundamentals of DA in benzonitrile are determined relative to the absolute Raman cross section of the respective solvent band appearing at $1000 \mathrm{~cm}^{-1}$.

\section{Simulation of the Raman excitation profiles (REPs) and the absorption spectra}

The absorption spectra and the absolute Raman excitation profiles for DA in acetonitrile and benzonitrile were modeled using Heller's time-dependent wave packet approach with the inclusion of solvent effects (Brownian oscillator model) as discussed in the section of theoretical and computational methods in order to estimate the vibrational and solvent reorganizational energies for the Franck-Condon excited $\left({ }^{1} \mathrm{CT}\right)$ state. The parameters required to carry out the simulation viz. the relative excited state distortions $\left(\Delta_{v}\right)$ of the normal coordinates in dimensionless units can be obtained from the knowledge of absolute Raman intensities $\left(I_{R}\right)$ and the frequencies of the normal vibrations $\left(\omega_{v}\right)$, obtained experimentally assuming, $I_{R} \propto \Delta_{v}^{2} \omega_{v}^{2}$. The zero-zero energy $\left(E_{0}\right)$ and the homogeneous linewidth $(\Gamma)$ is normally estimated from the absorption spectra and the low energy edge of the absorption, respectively. The value of the transition dipole moment $(M)$ is also estimated from the integrated area of the absorption spectra. In polar solvents, acetonitrile and benzonitrile that comprises of the underdamped oscillations due to the inertial response of the solvent, the knowledge of the oscillator frequency is required and these values are taken from Ref. 56.

The best fit parameters required to simulate the absolute resonance Raman intensities for the Raman active vibrations of DA in acetonitrile are presented in Table I along with their potential energy distributions (PEDs). The mode-specific vibrational reorganization energies calculated using Eq. (15) and other parameters, viz. zero-zero energy $\left(E_{0}\right.$ $\left.=18750 \mathrm{~cm}^{-1}\right)$, electronic transition dipole $(M=0.82 \AA)$, lifetime broadening $\left(\Gamma=90 \mathrm{~cm}^{-1}\right)$ used to fit the resonance Raman and absorption spectra are also included in the table. As observed from the mode-specific vibrational reorganization energies presented in the table, it is found that $27 \%$ of the total reorganization energy is involved in $\mathrm{N}-\mathrm{O}$ stretching vibration followed by $18 \%, 13 \%$, and $12 \%$ along $\mathrm{N}=\mathrm{N}$, $\mathrm{C}-\mathrm{C}$, and $\mathrm{C}-\mathrm{N}$ stretching vibrations. Thus, mostly the dynamics in the Franck-Condon excited state occurs along these vibrational modes. $30 \%$ of the remaining total vibrational reorganization energy is uniformly distributed among other vibrational modes of DA. The excitation profiles for the Raman active modes of DA in acetonitrile are shown in Fig. 5 along with the simulated curve. A good agreement between the experimental and the simulated curve is ob- 
TABLE I. Raman frequencies $\left(\omega_{v}\right.$ in $\left.\mathrm{cm}^{-1}\right)$, dimensionless displacements $\left(\Delta_{v}\right)$ in the resonant electronic state and the vibrational assignments of 4-nitro, $4^{\prime}$-dimethylamino-azobenzene in acetonitrile. $\nu$, stretch; $\delta$, in-plane bend. Zero-zero energy, $E_{0}=18750 \mathrm{~cm}^{-1}$; electronic transition dipole, $M$ $=0.82 \AA$; lifetime broadening, $\Gamma=90 \mathrm{~cm}^{-1}$.

\begin{tabular}{ccccl}
\hline \hline Mode & $\omega_{v} \mathrm{~cm}^{-1}$ & $\Delta_{v}$ & $\lambda_{v} \mathrm{~cm}^{-1}$ & $\begin{array}{l}\text { Potential energy } \\
\text { distributions }(\mathrm{PEDs})\end{array}$ \\
\hline$\nu_{8}$ & 1593 & 0.44 & 154.20 & $71 \nu(\mathrm{C}-\mathrm{C}), 18 \delta(\mathrm{C}-\mathrm{C})$ \\
$\nu_{7}$ & 1453 & 0.57 & 236.04 & $65 \nu(\mathrm{C}-\mathrm{C}), 10 \delta(\mathrm{C}-\mathrm{N})$ \\
$\nu_{6}$ & 1428 & 0.70 & 349.86 & $38 \nu(\mathrm{C}-\mathrm{C}), 22 \nu(\mathrm{N}-\mathrm{O})$, \\
& & & & $17 \delta(\mathrm{C}-\mathrm{N}), 10 \delta(\mathrm{C}-\mathrm{C})$ \\
$\nu_{5}$ & 1398 & 0.84 & 493.21 & $33 \nu(\mathrm{N}=\mathrm{N}), 19 \nu\left(\mathrm{C}_{\mathrm{Ph}}-\mathrm{N}_{\mathrm{Me}}\right)$, \\
& 1344 & 1.05 & 740.88 & $52 \nu(\mathrm{N}-\mathrm{O}), 32 \nu(\mathrm{C}-\mathrm{N})_{\mathrm{NO}}$ \\
$\nu_{4}$ & 1199 & 0.53 & 168.40 & $29 \nu(\mathrm{C}-\mathrm{C}), 26 \nu(\mathrm{C}-\mathrm{N})$, \\
$\nu_{3}$ & & & $23 \delta(\mathrm{C}-\mathrm{C}), 10 \delta(\mathrm{C}-\mathrm{H})$ \\
$\nu_{2}$ & 1138 & 0.76 & 328.65 & $30 \nu(\mathrm{C}-\mathrm{N}), 28 \delta(\mathrm{C}-\mathrm{C})$, \\
& & & & $21 \nu(\mathrm{C}-\mathrm{C})$ \\
$\nu_{1}$ & 1104 & 0.72 & 286.16 & $38 \nu(\mathrm{C}-\mathrm{C}), 34 \delta(\mathrm{C}-\mathrm{H})$, \\
& & & & $17 \nu(\mathrm{C}-\mathrm{N})_{\mathrm{NO}}$ \\
\hline
\end{tabular}

served. It is seen from the figure that the absolute Raman intensities closely follow the absorption spectral profile. In Fig. 6(a), we have shown the experimental (open bars) and simulated (solid bars) absolute Raman intensities for all the eight fundamental vibrations of DA in acetonitrile, for only some of the excitation wavelengths, 450, 480, 500, and 530 $\mathrm{nm}$, for brevity. A good agreement between the calculated and experimental absolute Raman intensities for all the vibrational modes at different excitation wavelengths confirm that the assumptions considered here are reasonable. The simulated absorption spectra (solid line) along with the ex-

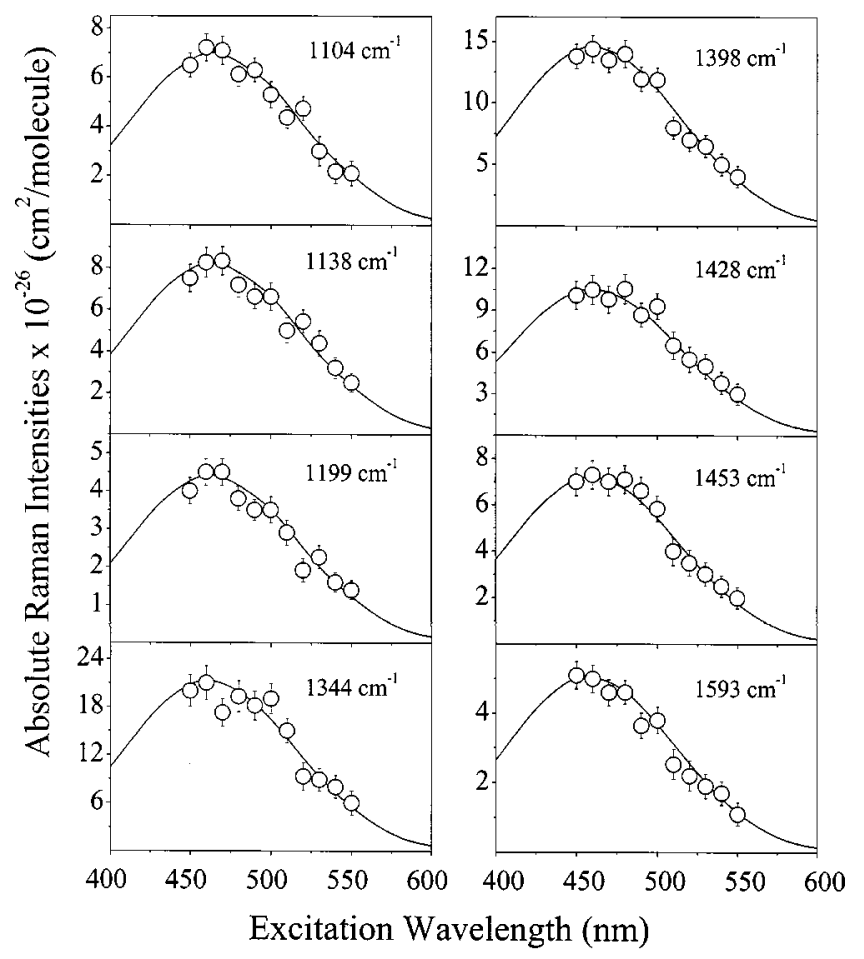

FIG. 5. Experimental (open circles) and simulated (solid line) Raman excitation profiles for $1104,1138,1199,1344,1398,1428,1453$, and $1593 \mathrm{~cm}^{-1}$ vibrations of DA in acetonitrile.

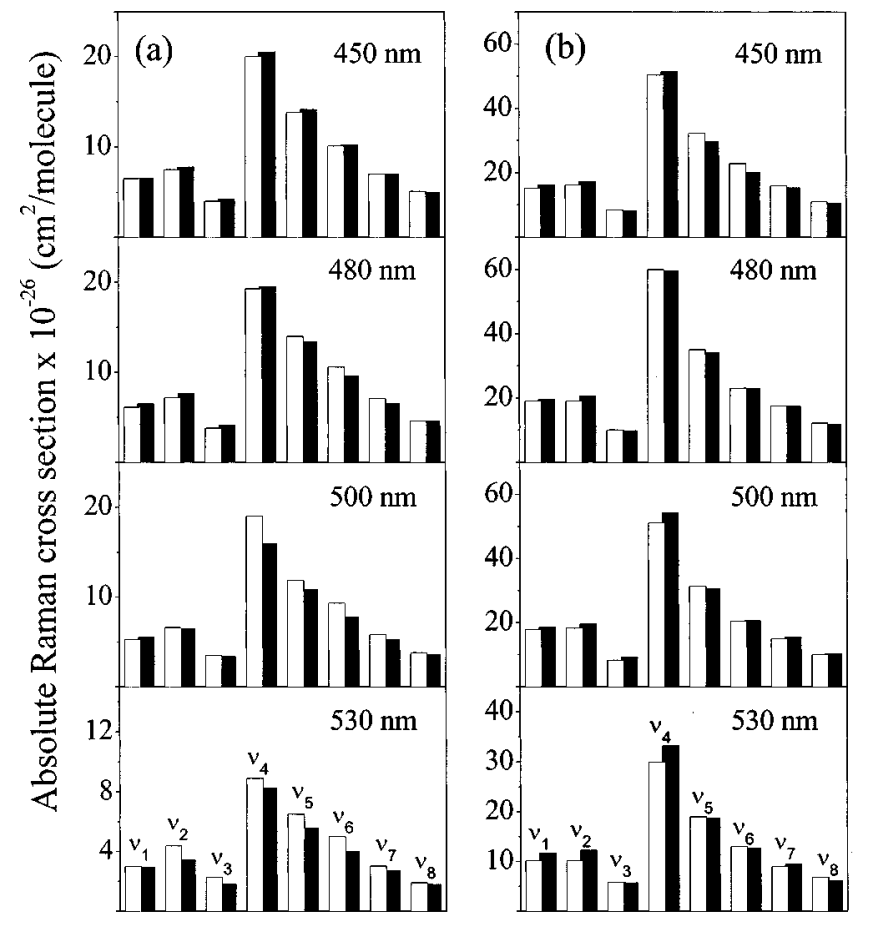

Vibrational Mode

FIG. 6. The experimental (open bars) and calculated (solid bars) absolute resonance Raman intensities of DA in (a) acetonitrile and (b) benzonitrile.

perimental absorption band for DA in acetonitrile are shown in Fig. 7(a). A reasonably good agreement between the calculated and the experimental curve is obtained.

For DA in benzonitrile, the best fit parameters required to simulate the absolute RR intensities and the absorption spectrum are given in Table II. The table includes the best fit values of dimensionless displacements corresponding to the normal vibrations of DA in benzonitrile along with their PEDs. The mode-specific vibrational reorganization energies

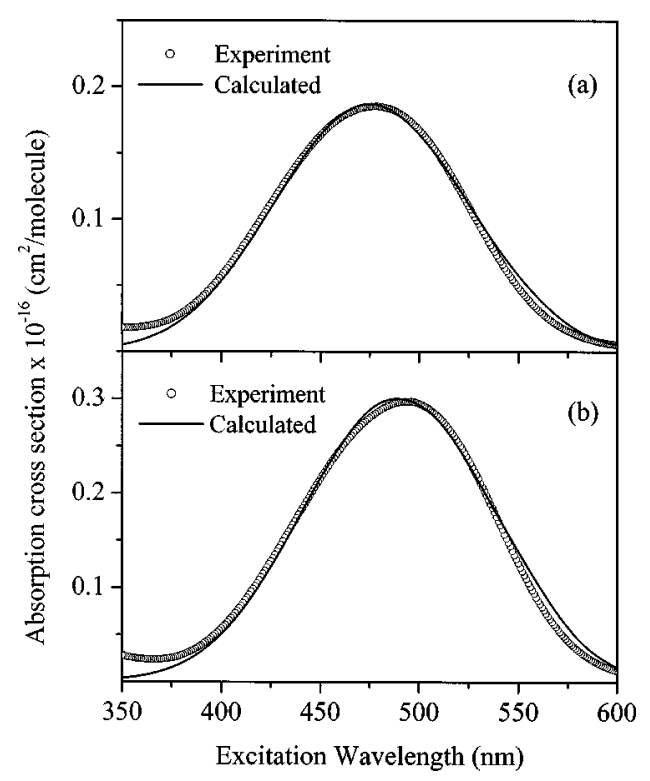

FIG. 7. Experimental (open circles) and calculated (solid line) absorption spectra of DA in (a) acetonitrile and (b) benzonitrile. 
TABLE II. Raman frequencies $\left(\omega_{v}\right.$ in $\left.\mathrm{cm}^{-1}\right)$, dimensionless displacements $\left(\Delta_{v}\right)$ in the resonant electronic state and the vibrational assignments of 4-nitro, $4^{\prime}$-dimethylamino-azobenzene in benzonitrile. $\nu$, stretch; $\delta$, in-plane bend. Zero-zero energy, $E_{0}=18350 \mathrm{~cm}^{-1}$; electronic transition dipole, $M$ $=1.02 \AA$; lifetime broadening, $\Gamma=60 \mathrm{~cm}^{-1}$.

\begin{tabular}{ccccl}
\hline \hline Mode & $\omega_{v} \mathrm{~cm}^{-1}$ & $\Delta_{v}$ & $\lambda_{v} \mathrm{~cm}^{-1}$ & $\begin{array}{c}\text { Potential energy } \\
\text { distributions }(\mathrm{PEDs})\end{array}$ \\
\hline$\nu_{8}$ & 1587 & 0.41 & 133.39 & $71 \nu(\mathrm{C}-\mathrm{C}), 18 \delta(\mathrm{C}-\mathrm{C})$ \\
$\nu_{7}$ & 1447 & 0.54 & 210.20 & $65 \nu(\mathrm{C}-\mathrm{C}), 10 \delta(\mathrm{C}-\mathrm{N})$ \\
$\nu_{6}$ & 1422 & 0.63 & 282.20 & $38 \nu(\mathrm{C}-\mathrm{C}), 22 \nu(\mathrm{N}-\mathrm{O})$, \\
& & & & $17 \delta(\mathrm{C}-\mathrm{N}), 10 \delta(\mathrm{C}-\mathrm{C})$ \\
$\nu_{5}$ & 1393 & 0.78 & 423.75 & $33 \nu(\mathrm{N}=\mathrm{N}), 19 \nu\left(\mathrm{C}_{\mathrm{Pb}}-\mathrm{N}_{\mathrm{Me}}\right)$, \\
& & & & $14 \nu(\mathrm{C}-\mathrm{C})$ \\
$\nu_{4}$ & 1336 & 1.07 & 764.79 & $52 \nu(\mathrm{N}-\mathrm{O}), 32 \nu(\mathrm{C}-\mathrm{N})_{\mathrm{NO}}$ \\
$\nu_{3}$ & 1195 & 0.48 & 137.66 & $29 \nu(\mathrm{C}-\mathrm{C}), 26 \nu(\mathrm{C}-\mathrm{N})$, \\
& & & & $23 \delta(\mathrm{C}-\mathrm{C}), 10 \delta(\mathrm{C}-\mathrm{H})$ \\
$\nu_{2}$ & 1140 & 0.73 & 303.75 & $30 \nu(\mathrm{C}-\mathrm{N}), 28 \delta(\mathrm{C}-\mathrm{C})$, \\
& & & & $21 \nu(\mathrm{C}-\mathrm{C})$ \\
$\nu_{1}$ & 1106 & 0.73 & 294.69 & $38 \nu(\mathrm{C}-\mathrm{C}), 34 \delta(\mathrm{C}-\mathrm{H})$, \\
& & & & $17 \nu(\mathrm{C}-\mathrm{N})_{\mathrm{NO}}$ \\
\hline \hline
\end{tabular}

as well as various fit parameters, viz. zero-zero energy, electronic transition dipole, lifetime broadening required for calculating the resonance Raman and absorption spectra are also included in the table. The mode-specific reorganization energy values of DA in benzonitrile as shown in Table II, reflect that upon vertical excitation of the solute from the ground electronic state to the Franck-Condon excited state, maximum reorganization occurs along $\mathrm{N}-\mathrm{O}$ stretch (30\%), followed by $\mathrm{N}=\mathrm{N}(17 \%)$ and $\mathrm{C}-\mathrm{N}(12 \%)$ stretches. Thus, the dynamics in the Franck-Condon excited state usually occurs along these vibrational modes. $41 \%$ of the remaining total vibrational reorganization energy is uniformly distributed among other normal vibrations of DA. The experimental and the simulated REPs for the eight Raman active modes of DA in benzonitrile are shown in Fig. 8. In Fig. 6(b) are shown the experimental (open bars) and simulated (solid bars) absolute Raman intensities for all the eight fundamental vibrations of DA in benzonitrile, for the excitation wavelengths, 450, 480, 500, and $530 \mathrm{~nm}$. A good agreement between the simulated and experimental absolute Raman intensities for DA in benzonitrile is observed for all the vibrational modes at the specified excitation wavelengths. As shown in Fig. 7(b), the simulated absorption spectra for DA in benzonitrile fit well with the respective experimental charge transfer absorption band of DA. For the simulation of absolute resonance Raman intensities in acetonitrile and benzonitrile, the concept of inertial and diffusive solvent relaxation are taken into consideration [Eqs. (8)-(12)]. In particular, the oscillator frequencies and diffusive solvent relaxation times for acetonitrile and benzonitrile are taken from Ref. 56.

\section{Short time dynamics}

Based on the simulation data, especially the dimensionless displacements of DA in acetonitrile and benzonitrile along various fundamental vibrations as shown in Tables I and II, the short time dynamics of DA under CT excitation can be inferred. As observed from the tables, the vibrational modes of DA in acetonitrile (benzonitrile), viz. 1344 (1336),

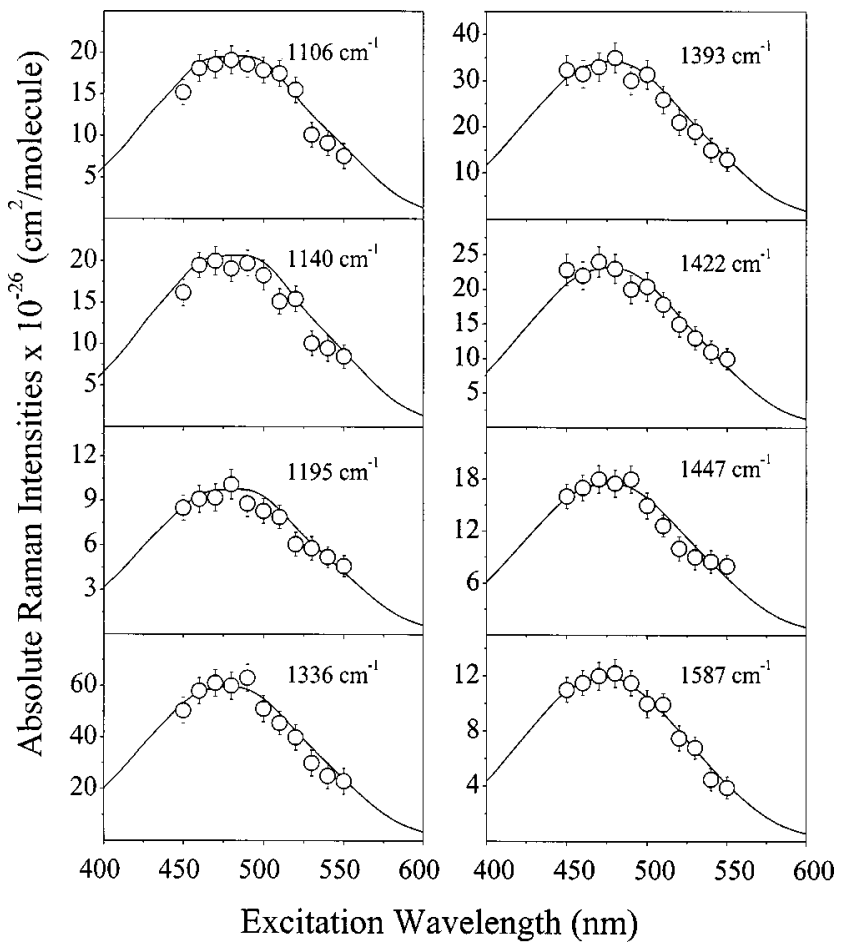

FIG. 8. Experimental (open circles) and simulated (solid line) Raman excitation profiles for $1106,1140,1195,1336,1393,1422,1447$, and $1587 \mathrm{~cm}^{-1}$ vibrations of DA in benzonitrile.

1398 (1393), 1138 (1140), and $1104(1106) \mathrm{cm}^{-1}$ have the largest displacements in the $\mathrm{CT}$ state. These modes are assigned to $\mathrm{N}-\mathrm{O}, \mathrm{N}=\mathrm{N}, \mathrm{C}-\mathrm{N}$, and $\mathrm{C}-\mathrm{C}$ stretches, respectively. It is possible to envisage such large displacements for these modes, when they participate in the charge transfer dynamics. Thus, in order to acquire further information about the short time dynamics, a detailed study of normal coordinate analysis using density functional theory (B3LYP) with $6-31 \mathrm{G}$ basis set is carried out for DA. ${ }^{65}$ Comparison of the experimental and theoretically calculated frequencies along with the calculated potential energy distributions (PEDs) for the observed fundamental vibrations are included in Tables I and II.

From the knowledge of the ground state normal mode description of DA at the equilibrium geometry, as well as the dimensionless displacements and employing Eqs. (16)-(18), the change in internal coordinates as a function of time is calculated. The changes at $20 \mathrm{fs}$ are only considered since the multimode correlation function decays within 20-30 fs. The RR intensity analysis only provides the magnitude but not the sign or direction of change of the FC active normal mode displacements. In reality, there are $2^{n}$ ( $n$ being the number of FC active modes) possible sign combinations that are equally consistent with the result of the RR intensity analysis. In the case of DA, there are $2^{8}$ different sign combinations possible for the normal mode displacements. Of these 256 possible combinations, many involve physically unreasonable bond length and bond angle changes and hence, can be easily ruled out. Among the remaining possibilities, we have selected those that involve the direction of the geometry change towards a zwitterionic structure as shown in Fig. 1. This specifically required the phenyl ( $\mathrm{Ph})$ 
TABLE III. Internal coordinate displacements of 4-nitro, $4^{\prime}$ dimethylamino-azobenzene at $20 \mathrm{fs}$ after photoexcitation.

\begin{tabular}{cccc}
\hline & \multirow{2}{*}{$\begin{array}{c}\text { Bond lengths and } \\
\text { bond angles (in } \AA \\
\text { Bonds }\end{array}$} & \multicolumn{2}{c}{ Time $(20 \mathrm{fs})$} \\
\cline { 3 - 4 } & 1.2886 & 0.0052 & Bcetonitrile \\
\hline $\mathrm{N}_{1} \mathrm{~N}_{2}$ & 1.3996 & -0.0071 & 0.0051 \\
$\mathrm{C}_{3} \mathrm{~N}_{1}$ & 1.4188 & -0.0001 & -0.0069 \\
$\mathrm{C}_{4} \mathrm{~N}_{2}$ & 1.4130 & 0.0035 & -0.0004 \\
$\mathrm{C}_{5} \mathrm{C}_{3}$ & 1.4112 & 0.0059 & 0.0033 \\
$\mathrm{C}_{6} \mathrm{C}_{4}$ & 1.3860 & -0.0008 & -0.0006 \\
$\mathrm{C}_{9} \mathrm{C}_{7}$ & 1.3912 & -0.0053 & -0.0006 \\
$\mathrm{C}_{10} \mathrm{C}_{8}$ & 1.3804 & -0.0028 & -0.0049 \\
$\mathrm{C}_{11} \mathrm{~N}_{21}$ & 1.4587 & -0.0273 & -0.0027 \\
$\mathrm{C}_{12} \mathrm{~N}_{22}$ & 1.2673 & 0.0307 & 0.0308 \\
$\mathrm{~N}_{22} \mathrm{O}_{33}$ & 1.2674 & 0.0309 & 0.0309 \\
$\mathrm{~N}_{22} \mathrm{O}_{34}$ & 116.5 & 0.0012 & 0.0011 \\
$\mathrm{C}_{3} \mathrm{~N}_{1} \mathrm{~N}_{2}$ & 114.9 & 0.0001 & 0.0001 \\
$\mathrm{C}_{4} \mathrm{~N}_{2} \mathrm{~N}_{1}$ & & & \\
\hline \hline
\end{tabular}

${ }^{\mathrm{a} C a l c u l a t e d}$ from B3LYP/6-31G.

rings to change towards quinoidal structure and also the $\mathrm{N}=\mathrm{N}$ and $\mathrm{N}-\mathrm{O}$ bonds to lengthen and $\mathrm{C}(\mathrm{Ph})-\mathrm{N}$ bonds to shorten. Only one of the possible sign combinations gave a realistic change in geometry and hence was taken as the most probable combination. Hence, taking the latter sign combination for the dimensionless displacements of the observed normal vibrations we have carried out the time-dependent structural dynamics of DA in acetonitrile and benzonitrile. In Table III, we have reported the changes in $\mathrm{N}_{1}=\mathrm{N}_{2}, \mathrm{C}_{3}-\mathrm{N}_{1}$, $\mathrm{C}_{4}-\mathrm{N}_{2}, \mathrm{C}_{5}-\mathrm{C}_{3}, \mathrm{C}_{6}-\mathrm{C}_{4}, \mathrm{C}_{9}-\mathrm{C}_{7}, \mathrm{C}_{10}-\mathrm{C}_{8}, \mathrm{C}_{11}-\mathrm{N}_{21}$, $\mathrm{C}_{12}-\mathrm{N}_{22}, \mathrm{~N}_{22}-\mathrm{O}_{33}$, and $\mathrm{N}_{22}-\mathrm{O}_{34}$ bond lengths and $\mathrm{C}_{3}-\mathrm{N}_{1}-\mathrm{N}_{2}$ and $\mathrm{C}_{4}-\mathrm{N}_{2}-\mathrm{N}_{1}$ bond angles of DA in acetonitrile and benzonitrile at $20 \mathrm{fs}$ after photoexcitation. The bond lengths ${ }^{65}$ in $\AA$ and bond angles in degrees calculated from B3LYP with 6-31G basis set are also included in the table. It is observed from the table that the changes involved in DA within 20 fs along $\mathrm{N}=\mathrm{N}, \mathrm{C}_{3}-\mathrm{N}_{1}$, and $\mathrm{C}-\mathrm{C}(\mathrm{Ph})$ stretching vibrations are of the order of $0.005 \AA$, while those along $\mathrm{C}_{12}-\mathrm{N}_{22}$ and $\mathrm{N}-\mathrm{O}$ bonds are of the order of $0.03 \AA$. Thus, from the short time dynamics, it can be inferred that the $\mathrm{N}=\mathrm{N}, \mathrm{C}-\mathrm{N}, \mathrm{C}-\mathrm{C}$, and $\mathrm{N}-\mathrm{O}$ stretching vibrations experience considerable amount of distortion immediately upon photoexcitation to the CT state and evolve towards a zwitterionic structure. The short time dynamics is found to be very similar in the two solvents, acetonitrile and benzonitrile.

\section{DISCUSSION}

In order to carry out a comparative study of the results obtained in polar solvents having different relaxation behavior, we present in Table IV the experimental and calculated absolute Raman cross sections for the eight fundamental vibrations of DA in acetonitrile and benzonitrile $\left(\lambda_{\operatorname{exc}}\right.$ $=490 \mathrm{~nm}$ ). From the table it is observed that the experimental and calculated absolute Raman intensities fit reasonably well for all the vibrational modes in the two solvents.

The vibrational and solvent reorganization energies of DA in acetonitrile and benzonitrile are presented in Table V. From our results, we can summarize that in both the solvents, acetonitrile and benzonitrile, the vibrational reorgani-
TABLE IV. Experimental and calculated absolute Raman cross sections of 4-nitro, $4^{\prime}$-dimethylamino-azobenzene in acetonitrile and benzonitrile at 490 $\mathrm{nm}$.

\begin{tabular}{|c|c|c|c|c|c|}
\hline \multicolumn{3}{|c|}{ Acetonitrile } & \multicolumn{3}{|c|}{ Benzonitrile } \\
\hline & \multicolumn{2}{|c|}{$\begin{array}{l}\text { Absolute Raman } \\
\text { cross section } \\
\times 10^{-26} \\
\left(\mathrm{~cm}^{2} / \text { molecule }\right)\end{array}$} & \multirow[b]{2}{*}{$\omega_{v} \mathrm{~cm}^{-1}$} & \multicolumn{2}{|c|}{$\begin{array}{l}\text { Absolute Raman } \\
\text { cross section } \\
\times 10^{-26} \\
\left(\mathrm{~cm}^{2} / \text { molecule }\right)\end{array}$} \\
\hline$\omega_{v} \mathrm{~cm}^{-1}$ & Expt. & Calc. & & Expt. & Calc. \\
\hline 1593 & 3.65 & 4.11 & 1587 & 11.50 & 11.22 \\
\hline 1453 & 6.62 & 6.01 & 1447 & 18.00 & 16.87 \\
\hline 1428 & 8.69 & 8.83 & 1422 & 20.00 & 22.33 \\
\hline 1398 & 11.95 & 12.30 & 1393 & 30.00 & 33.11 \\
\hline 1344 & 18.14 & 18.05 & 1336 & 63.00 & 58.18 \\
\hline 1199 & 3.50 & 3.81 & 1195 & 8.80 & 9.68 \\
\hline 1138 & 6.62 & 7.17 & 1140 & 19.73 & 20.59 \\
\hline 1104 & 6.30 & 6.10 & 1106 & 18.59 & 19.50 \\
\hline
\end{tabular}

zation energies for DA are distributed among various intramolecular vibrations, being maximum for the $\mathrm{N}-\mathrm{O}$ stretch, followed by the $\mathrm{N}=\mathrm{N}$ stretch. These normal vibrations are strongly coupled to the CT transition and hence, undergo large resonant excited state displacements and thus, contribute to the vibrational reorganization energy. It is also observed from Table $\mathrm{V}$ that the total vibrational reorganization energy is almost similar in magnitude for DA in acetonitrile $\left(\lambda_{v}=2757 \mathrm{~cm}^{-1}\right)$ and benzonitrile $\left(\lambda_{v}=2551 \mathrm{~cm}^{-1}\right)$ indicating the fact that structural distortions are almost similar in both these solvents. Similar structural distortions along various normal coordinates of DA in the two solvents, acetonitrile and benzonitrile result in similar short time dynamics (as shown in Table III) leading to a zwitterionic structure within 20 fs after photoexcitation.

The total solvent reorganization energy term has been partitioned into its inertial and diffusive counterparts in acetonitrile and benzonitrile. It is important to separate these two solvent reorganization energy terms since it is useful in calculating the electron transfer rates. Since acetonitrile is known to be a fast solvent and can respond in ultrafast time scale to the changes in solute charge distribution, it can be assumed to have a greater magnitude for the reorganization energy component arising due to inertial relaxation in comparison to the slow (arising due to diffusion) relaxation component. But it is reverse in benzonitrile that is more viscous than acetonitrile and is known to be a slow solvent. Benzonitrile responds rather slowly to the changes in solute charge distribution as a result of which one can assume maximum contribution to relaxation occurs via diffusion and not by the

TABLE V. Total vibrational $\left(\lambda_{v}\right)$ and solvent $\left(\lambda_{s}\right)$ reorganization energies of 4-nitro, $4^{\prime}$-dimethylamino-azobenzene in acetonitrile and benzonitrile.

\begin{tabular}{ccc}
\hline & Acetonitrile & Benzonitrile \\
\hline$\pi^{*}$ & 0.713 & 0.904 \\
$\lambda_{v}\left(\mathrm{~cm}^{-1}\right)$ & 2757 & 2551 \\
$\lambda_{s}\left(\mathrm{~cm}^{-1}\right)$ & 1600 & 1520 \\
$\lambda_{I}\left(\mathrm{~cm}^{-1}\right)$ & 1150 & 680 \\
$\lambda_{D}\left(\mathrm{~cm}^{-1}\right)$ & 450 & 840 \\
\hline \hline
\end{tabular}


inertial motion. Thus, $\lambda_{I}$ and $\lambda_{D}$ are found to be the best fit parameters obtained from our simulations for the absorption spectra and Raman excitation profiles. For the simulations the inertial reorganization energies are varied over a range from $1300-1000$ and $800-500 \mathrm{~cm}^{-1}$ in acetonitrile and benzonitrile, respectively. Correspondingly, the diffusive reorganization energies vary over a range from 300-600 and 700$1000 \mathrm{~cm}^{-1}$ in acetonitrile and benzonitrile. Very low and high values for the reorganization energies result in structured and broad REPs, respectively. In acetonitrile, the best fit values for $\lambda_{I}$ and $\lambda_{D}$ are 1150 and $450 \mathrm{~cm}^{-1}$ as observed in Table $\mathrm{V}$ while in benzonitrile, the best fit values for $\lambda_{I}$ and $\lambda_{D}$ are 680 and $840 \mathrm{~cm}^{-1}$, respectively. The diffusive solvent relaxation energies ${ }^{56}\left(\Lambda_{D}\right)$ correspond to 52.9 and $6.3 \mathrm{~cm}^{-1}$ in acetonitrile and benzonitrile and the oscillator frequencies $(\omega)$ correspond to 375 and $95.2 \mathrm{~cm}^{-1}$ in acetonitrile and benzonitrile, respectively.

From the knowledge of the total vibrational and solvent reorganization energies of DA in the polar solvents, acetonitrile as well as benzonitrile, it is found that in highly polar solvents, the vibrational reorganization energy has considerable magnitude suggesting that in polar solvents, photoexcitation to the FC excited state is followed by a large rearrangement of the intramolecular vibrations of the solute and thus, significant contribution from $\lambda_{v}$ is observed in these solvents. In polar solvents, the response of the ultrafast inertial component in addition to the slow diffusive component has been studied. From the results as shown in Table V, a reasonable estimate of the solvent reorganization energy terms (viz. inertial and diffusive) has been made. It is assumed that in fast solvent, acetonitrile, the inertial solvent reorganization energy $\left(\lambda_{I}\right)$ dominates over the slow diffusive reorganization energy $\left(\lambda_{D}\right)$. Hence, for ultrafast electron transfer reactions one can use the inertial reorganization energy term instead of the total solvent reorganization. This is in contrast to slow solvents like benzonitrile where the diffusive reorganization energy term dominates over the ultrafast inertial reorganization energy.

\section{CONCLUSIONS}

In the present study, Raman measurements have been carried out in resonance with the charge transfer transition of DA in two solvents of different polarity, viz. acetonitrile and benzonitrile using excitation wavelengths ranging from 450$550 \mathrm{~nm}$. We have used Heller's wave packet dynamical approach with the inclusion of solvent that is treated as a Brownian oscillator to simulate the absorption spectra and the absolute resonance Raman excitation profiles of DA in the two solvents specified above. There are a few limitations in these calculations arising from the unknown excited state vibrational frequencies and the low frequency molecular motions that are normally lumped into the solvent reorganization energy term. Additional limitations arise due to the neglect of mixing of the normal coordinates (Duschinsky rotation) and coordinate dependence of the transition dipole moment and assuming harmonic ground and excited state potential energy surfaces. These limitations are not expected to lead to serious errors in the calculations because, (a) our interest lies only in the Franck-Condon region of the poten- tial energy surface, and (b) this approach provides a good estimate for the different contribution of solvent reorganization energies as well as mode-specific vibrational reorganization energies (calculated from the dimensionless displacements for the respective normal vibrations). In the solvents acetonitrile and benzonitrile, we have partitioned the total solvent reorganization energy to its inertial and diffusive counterparts. The results in both the solvents infer that the total vibrational reorganization energy has a large magnitude indicating more structural distortions in the FC excited state in these solvents. The vibrational reorganization energy is distributed among various intramolecular vibrations, being maximum for the $\mathrm{N}-\mathrm{O}$ stretch, followed by the $\mathrm{N}=\mathrm{N}$ stretch in both the solvents. The total solvent reorganization energy is partitioned into its inertial and diffusive components in the two solvents, acetonitrile and benzonitrile. Acetonitrile being a fast solvent is assumed to have a larger contribution from the inertial component than its diffusive counterpart while the diffusive reorganization energy has a greater magnitude as compared to the inertial reorganization energy in benzonitrile that is a slow solvent. In order to visualize the short time dynamics of DA in the two solvents, in terms of bond length and bond angle changes, the dimensionless displacements obtained from our simulations are converted with a priori knowledge of the accurate normal mode analysis. The short time dynamics in DA infers that within 20 fs after photoexcitation, the excited state evolution occurs along $\mathrm{N}-\mathrm{O}, \mathrm{N}=\mathrm{N}, \mathrm{C}-\mathrm{N}$, and $\mathrm{C}-\mathrm{C}$ stretching vibrations.

\section{ACKNOWLEDGMENTS}

The authors would like to thank Professor Biman Bagchi for useful suggestions. We thank the Council of Scientific and Industrial Research, the Department of Science and Technology and Jawaharlal Nehru Center for Advanced Scientific Research, Government of India for financial assistance and the Supercomputer Education and Research Center of Indian Institute of Science for providing the computing facilities necessary to carry out the present work.

\footnotetext{
${ }^{1}$ J. Herbich and A. Kapturkiewicz, J. Am. Chem. Soc. 120, 1014 (1998).

${ }^{2}$ P. F. Barbara, T. J. Meyer, and M. A. Ratner, J. Phys. Chem. 100, 13148 (1996).

${ }^{3}$ K. Wynne, C. Galli, and R. M. Hochstrasser, J. Chem. Phys. 100, 4797 (1994).

${ }^{4}$ A. B. Myers, Chem. Phys. 180, 215 (1994); Chem. Rev. 96, 911 (1996); J. Raman Spectrosc. 28, 389 (1997); Acc. Chem. Res. 30, 519 (1997).

${ }^{5}$ N. E. Koenig, T. Kuehne, D. Schwarzer, P. Voehringer, and J. Schroeder, Chem. Phys. Lett. 253, 69 (1996).

${ }^{6}$ S. Roy and B. Bagchi, J. Phys. Chem. 98, 9207 (1994).

${ }^{7}$ B. B. Smith, A. Staib, and J. T. Hynes, Chem. Phys. 176, 521 (1993).

${ }^{8}$ P. F. Barbara, G. C. Walker, and T. P. Smith, Science 256, 975 (1992).

${ }^{9}$ S. K. Doorn, R. L. Blackbourn, C. S. Johnson, and J. T. Hupp, Electrochim. Acta 36, 1775 (1991).

${ }^{10}$ P. J. Reid and P. F. Barbara, J. Phys. Chem. 99, 3554 (1995); K. Yoshihara, K. Tominaga, and Y. Nagasawa, Bull. Chem. Soc. Jpn. 68, 696 (1995); D. Braun, P. L. Nordio, A. Polimeno, and G. Saielli, Chem. Phys. 208, 127 (1996).

${ }^{11}$ P. F. Barbara and W. Jarzeba, Adv. Photochem. 15, 1 (1990); M. Kosower and D. Huppert, Annu. Rev. Phys. Chem. 37, 127 (1986); G. R. Fleming and M. Cho, ibid. 47, 109 (1996).

${ }^{12}$ T. J. Kang, W. Jarzeba, and P. F. Barbara, Chem. Phys. 149, 81 (1990); M.
} 
Cho, J.-Y. Yu, T. Joo, Y. Nagasawa, S. A. Passino, and G. R. Fleming, J. Phys. Chem. 100, 11944 (1996); T. Joo, Y. Jia, J.-Y. Yu, M. J. Lang, and G. R. Fleming, J. Chem. Phys. 104, 6089 (1996).

${ }^{13}$ M. Maroncelli, J. MacInnis, and G. R. Fleming, Science 243, 1674 (1989); R. S. Fee, J. A. Milsom, and M. Maroncelli, J. Phys. Chem. 95, 5170 (1991); M. Maroncelli and G. R. Fleming, J. Chem. Phys. 86, 6221 (1987); E. W. Castner, M. Maroncelli, and G. R. Fleming, ibid. 86, 1090 (1987).

${ }^{14}$ (a) F. Markel, N. S. Ferris, I. R. Gould, and A. B. Myers, J. Am. Chem. Soc. 114, 6208 (1992); (b) B. Li, A. E. Johnson, S. Mukamel, and A. B. Myers, ibid. 116, 11039 (1994); (c) A. B. Myers and B. Li, Chem. Phys. 92, 3310 (1990); (d) D. L. Phillips and A. B. Myers, J. Chem. Phys. 95, 226 (1991); (e) F. Markel and A. B. Myers, ibid. 98, 21 (1993).

${ }^{15}$ K. Kulinowski, I. R. Gould, N. S. Ferris, and A. B. Myers, J. Phys. Chem. 99, 17715 (1995).

${ }^{16}$ K. Kulinowski, I. R. Gould, and A. B. Myers, J. Phys. Chem. 99, 9017 (1995).

${ }^{17}$ J. D. Simon and S.-G. Su, J. Phys. Chem. 92, 2395 (1988); S.-G. Su and J. D. Simon, J. Chem. Phys. 89, 908 (1988); J. D. Simon and S.-G. Su, Chem. Phys. 152, 143 (1991).

${ }^{18}$ S. Mukamel, Principles of Nonlinear Optical Spectroscopy (Oxford University Press, New York, 1995).

${ }^{19}$ Y. J. Yan, M. Sparpaglione, and S. Mukamel, J. Phys. Chem. 92, 4842 (1988); M. Sparpaglione and S. Mukamel, J. Chem. Phys. 88, 3263 (1988).

${ }^{20}$ H. J. Kim and J. T. Hynes, J. Chem. Phys. 93, 5194 (1990); 93, 5211 (1990).

${ }^{21}$ H. Sumi and R. A. Marcus, J. Chem. Phys. 84, 4894 (1986); W. Nadler and R. A. Marcus, ibid. 86, 3906 (1987).

${ }^{22}$ D. F. Calef and P. G. Wolynes, J. Chem. Phys. 78, 470 (1983); P. G. Wolynes, ibid. 86, 5133 (1987)

${ }^{23}$ M. Maroncelli and G. R. Fleming, J. Chem. Phys. 89, 5044 (1988); M. Cho and R. J. Silbey, ibid. 103, 595 (1995)

${ }^{24}$ B. Bagchi and N. Gayathri, Adv. Chem. Phys. 107, 1 (1999); S. Roy and B. Bagchi, J. Chem. Phys. 99, 1310, 9938 (1993).

${ }^{25}$ W. M. Kwok, D. L. Phillips, P. K.-Y. Yeung, and V. W.-W. Yam, J. Phys. Chem. A 101, 9286 (1997); Chem. Phys. Lett. 262, 699 (1996).

${ }^{26}$ S. K. Doorn and J. T. Hupp, J. Am. Chem. Soc. 111, 4704 (1989); 111, 1142 (1989); R. D. Williams, V. I. Petrov, H. P. Lu, and J. T. Hupp, J. Phys. Chem. A 101, 8070 (1997); R. L. Blackbourn, C. S. Johnson, J. T. Hupp, M. A. Bryant, R. L. Sobocinski, and J. E. Pemberton, J. Phys. Chem. 95, 10535 (1991); S. K. Doorn, R. L. Blackbourn, C. S. Johnson, and J. T. Hupp, Electrochim. Acta 36, 1775 (1991).

${ }^{27}$ R. A. Marcus and N. Sutin, Biochim. Biophys. Acta 811, 265 (1985).

${ }^{28}$ R. A. Marcus, J. Chem. Phys. 24, 966 (1956).

${ }^{29}$ R. A. Marcus, Annu. Rev. Phys. Chem. 15, 155 (1964).

${ }^{30}$ N. Biswas and S. Umapathy, Chem. Phys. Lett. 234, 24 (1995).

${ }^{31}$ N. Biswas and S. Umapathy, J. Chem. Phys. 107, 7849 (1997); N. Biswas, B. Abraham, and S. Umapathy, J. Phys. Chem. A 106, 9397 (2002).

${ }^{32}$ M. K. Lawless and R. A. Mathies, J. Chem. Phys. 100, 2492 (1994); M. K. Lawless, S. D. Wickham, and R. A. Mathies, J. Am. Chem. Soc. 116, 1593 (1994); P. J. Reid, A. P. Shreve, and R. A. Mathies, J. Phys. Chem. 97, 12691 (1993).

${ }^{33}$ (a) X. Ci and A. B. Myers, J. Chem. Phys. 96, 6433 (1992); (b) A. B. Myers and B. Li, ibid. 92, 3310 (1990); (c) X. Ci, M. A. Pereira, and A. B. Myers, ibid. 92, 4708 (1990); (d) A. B. Myers, B. Li, and X. Ci, ibid. 89, 1876 (1988).

${ }^{34}$ A. B. Myers and R. A. Mathies, in Biological Applications of Raman Spectroscopy, edited by T. G. Spiro (Wiley, New York, 1987), Vol. 2, p. 1.

${ }^{35}$ J. I. Zink and K.-S. K. Shin, Advances in Photochemistry (Wiley, New York, 1991), Vol. 16, p. 119.
${ }^{36}$ A. B. Myers, in Laser Techniques in Chemistry, edited by A. B. Myers and T. R. Rizzo (Wiley, New York, 1995), Vol. 23, p. 325.

${ }^{37}$ (a) N. Biswas, S. Umapathy, C. Kalyanaraman, and N. Sathyamurthy, Proc.-Indian Acad. Sci., Chem. Sci. 107, 233 (1995); (b) N. Biswas and S. Umapathy, Pramana, J. Phys. 48, 937 (1997); (c) N. Biswas, Ph.D. dissertation, Indian Institute of Science, Bangalore, India, 1998.

${ }^{38}$ N. Biswas and S. Umapathy, Chem. Phys. Lett. 294, 181 (1998).

${ }^{39}$ A. M. Moran, C. Delbecque, and A. M. Kelley, J. Phys. Chem. A 105, 10208 (2001).

${ }^{40}$ A. M. Moran, D. S. Egolf, M. Blanchard-Desce, and A. M. Kelley, J. Chem. Phys. 116, 2542 (2002).

${ }^{41}$ A. M. Moran, M. Blanchard-Desce, and A. M. Kelley, Chem. Phys. Lett. 358, 320 (2002)

${ }^{42}$ A. M. Moran, G. P. Bartholomew, G. C. Bazan, and A. M. Kelley, J. Phys. Chem. A 106, 4928 (2002).

${ }^{43}$ X. Cao and J. L. McHale, J. Chem. Phys. 109, 1901 (1998).

${ }^{44}$ J. L. McHale, Acc. Chem. Res. 34, 265 (2001).

${ }^{45}$ F. Terenziani, A. Painelli, and D. Comoretto, J. Phys. Chem. A 104, 11049 (2000).

${ }^{46}$ T. Yamaguchi, Y. Kimura, and N. Hirota, J. Chem. Phys. 109, 9075 (1998).

${ }^{47}$ R. Jimenez, G. R. Fleming, P. V. Kumar, and M. Maroncelli, Nature (London) 369, 471 (1994)

${ }^{48}$ S. J. Rosenthal, X. Xie, M. Du, and G. R. Fleming, J. Chem. Phys. 95, 4715 (1991)

${ }^{49}$ E. W. Castner, Jr., M. Maroncelli, and G. R. Fleming, J. Chem. Phys. 86, 1090 (1987)

${ }^{50}$ S. A. Passino, Y. Nagasawa, and G. R. Fleming, J. Chem. Phys. 107, 6094 (1997).

${ }^{51}$ T. Lian, Y. Kholodenko, and R. M. Hochstrasser, J. Phys. Chem. 99, 2546 (1995).

${ }^{52}$ M. Maroncelli, J. Chem. Phys. 94, 2084 (1991).

${ }^{53}$ Y. Tanimura and S. Mukamel, J. Opt. Soc. Am. B 10, 2263 (1993).

${ }^{54}$ J. O. Morley, J. Chem. Soc., Faraday Trans. 91, 2067 (1995).

${ }^{55}$ J. M. Kamlet, J.-L. M. Abboud, and R. W. Taft, J. Am. Chem. Soc. 99, 6027 (1977).

${ }^{56}$ M. L. Horng, J. A. Gardecki, A. Papazyan, and M. Maroncelli, J. Phys. Chem. 99, 17311 (1995).

${ }^{57}$ A. Ulman, C. S. Willand, W. Koehler, D. R. Robello, D. J. Williams, and L. Handley, J. Am. Chem. Soc. 112, 7083 (1990).

${ }^{58}$ J. D. Womack, C. K. Mann, and T. J. Vickers, Appl. Spectrosc. 43, 527 (1989).

${ }^{59}$ N. Biswas and S. Umapathy, Appl. Spectrosc. 52, 496 (1998).

${ }^{60}$ J. M. Dudik, C. R. Johnson, and S. A. Asher, J. Chem. Phys. 82, 1732 (1985)

${ }^{61}$ (a) S.-Y. Lee and E. J. Heller, J. Chem. Phys. 71, 4777 (1979); (b) E. J. Heller, ibid. 62, 1544 (1975); (c) E. J. Heller, ibid. 68, 2066 (1978); (d) K. C. Kulander and E. J. Heller, ibid. 69, 2439 (1978); (e) E. J. Heller, Acc. Chem. Res. 14, 368 (1981); (f) A. B. Myers, R. A. Mathies, D. J. Tannor, and E. J. Heller, J. Chem. Phys. 77, 3857 (1982).

${ }^{62}$ M. D. Feit, J. A. Fleck, Jr., and A. Steiger, J. Comput. Phys. 47, 412 (1982).

${ }^{63}$ R. Kosloff, J. Phys. Chem. 92, 2087 (1988); S. O. Williams and D. G. Imre, 92, 3363 (1988).

${ }^{64}$ E. B. Wilson, Jr., J. C. Decius, and P. C. Cross, Molecular Vibrations (Dover, New York, 1980).

${ }^{65}$ N. Biswas and S. Umapathy, J. Phys. Chem. A 104, 2734 (2000).

${ }^{66}$ M. J. Frisch et al., GAUSSIAN 94, Revision C.2, Gaussian, Inc., Pittsburgh, PA, 1995.

${ }^{67}$ (a) N. Biswas and S. Umapathy, J. Phys. Chem. A 101, 5555 (1997); (b) P. Mohandas and S. Umapathy, ibid. 101, 4449 (1997). 